\title{
Primeros datos sobre el arroyo B de Marroquíes Bajos (Jaén)
}

Juana Cano Carrillo*

Durante los meses de Agosto a Diciembre de 1995 se efectuó una excavación de urgencia en el yacimiento arqueológico de Marroquíes Bajos, concretamente en la Unidad de Actuación 23, manzana B, parcela 3-4 (Figs. I y 2). En el momento de inicio de los trabajos el solar ya había sido rebajado con medios mecánicos hasta la base geológica e incluso también se había rebajado parte del sustrato geológico, de modo que la única estratigrafía que se pudo documentar correspondía a los silos y fosos de la Edad del Cobre y del Bronce. Por este motivo, a excepción del nivel de arrroyada, los demás elementos medievales aquí reflejados sólo se pudieron estudiar por la documentación de algunos de los perfiles del solar y por la excavación posterior de la parcela destinada a zonas comunes, como patio, piscina, etc, aún intacta y situada al este del solar, en la que sí se pudo constatar la presencia y trazado de un arroyo de origen medieval y con funcionamiento a lo largo del tiempo hasta épocas modernas. En conclusión, los dos elementos que nos proporcionan más información corresponden al arroyo y al perfil Norte del solar.

El arroyo atraviesa el solar en dirección sureste-noroeste y en la mayor parte de su trazado, al menos lo que se conservaba y fue posible documentar, aprovechaba el recorrido de uno de los fosos del poblado de la Edad del Cobre, de tal modo que en muchas zonas rompía los niveles de colmatación calcolítica del foso ocupando buena parte del mismo (Fig. 3). El material que encontramos en los niveles de arroyada era de gran interés porque, a pesar de que la mayor parte del mismo se encontraba muy erosionado y era de arrastre, también se pudieron recoger gran cantidad de elementos de alfar, de piezas completas pero con algún defecto de fabricación, y al menos tres candiles, casi todo ello correspondiente al pe-ríodo almohade, aún cuando también se encontraron en los niveles de arroyada cerámicas incluso de época contemporánea, lo cual nos indica que el arroyo estuvo funcionando hasta fechas muy recientes. Cabe decir asimismo que el arroyo presentaba bifurcaciones en muchos puntos de su recorrido.

Sin embargo, quizás más interesante que el propio trazado del arroyo sea el análisis del perfil Norte de la parcela por la clara secuencia estratigráfica que nos proporciona el análisis del mismo (Fig. 4). En él es posible apreciar perfectamente la existencia de una estructura de tapial de casi cuatro metros de longitud, con una caida en horizontal. Dicho muro se levanta sobre un alzado natural de la roca y junto a él se distingue uno de los brazos de la arroyada mencionados anteriormente. Tal y como se puede ver en la figura 2, el perfil muestra un total de 10 unidades estratigráficas y los niveles se encuentran bastante horizontalizados.

* Arqueóloga Profesional 
El primer estrato se corresponde con los niveles de relleno y colmatación del terreno creados en época contemporánea, rellenos que se definen por una tierra muy oscura, de cultivo, que en muchos casos se encuentra mezclada con escombreras.

Bajo él se encuentra el nivel del arroyo, que se extiende por toda la sección del perfil. En él encontramos gran cantidad de tejas, cantos rodados, restos de fauna de todo tipo, etc. Aunque aparecen gran número de elementos cerámicos, el estado de erosión en el que se encuentra hace difícil definir su adscripción cultural. Sin embargo, sí podemos señalar que la mayor parte del mismo parece de época almohade. La U.S. 6 es otra de las bifurcaciones o ramales del mismo arroyo, aunque pudiera haberse formado con anterioridad.

El tercer estrato se corresponde con un nivel de arena perteneciente a los depósitos del arroyo y en la que no se pudo recuperar ningún elemento cerámico.

El muro de tapial caído se corresponde con la unidad estratigráfica 5 , se trata de una estructura de tapial de calicanto de unas dimensiones aproximadas de casi cuatro metros de largo por unos $60 \mathrm{~cm}$. de espesor, con una caída en horizontal.

Este muro está asociado al estrato 4 formado por un conjunto de piedras labradas más o menos regularmente, que por su situación con respecto al muro nos induce a pensar en la posibilidad de que fueran las cimentación del mismo, esto se avala por el hecho de estar excavado junto a él una fosa (U.S.7) en la que el material predominante eran tejas, sin embargo, el conjunto de elementos cerámicos que se recuperaron no eran lo suficientemente significativos para fecharnos el muro.

Bajo el muro de tapial hay un nivel de derrumbe de tejas correspondiente también a la caída del derrumbe de la construcción.

Tanto el estrato 9 como el 10 pertenecen ya al estratigrafía calcolítica de las estructuras documentadas en el solar, de hecho la U.S. 10 se compone de parte de los niveles del foso excavados en la base geológica

Continuamente se cita en las fuentes escritas la abundancia de los recursos hídricos de la ciudad de Jaén. Por otra parte, las numerosas excavaciones tanto en la zona de Marroquíes Bajos como en el interior del casco histórico han evidenciado la potente y extensa capa freática existente en nuestra ciudad, abundancia que se debe al privilegiado emplazamiento de la ciudad en la cima de un gran macizo, cuya composición geológica da lugar a la formación de la misma.

Algunas de las excavaciones en curso han puesto de manifiesto la existencia en la zona de un poblamiento rural disperso aunque de gran entidad desde época emiral. Para nosotros es difícil fechar los elementos encontrados sin una excavación que avale nuestras hipótesis, pero podemos aventurar que el arroyo se formó en el período almohade, puesto que apenas se han encontrado cerámicas medievales anteriores a esa fase. Es de destacar el hecho de que el arroyo aprovecha en gran medida el "cauce natural" proporcionado por el trazado del foso calcolítico, y el hecho de que luego se situe por encima del muro, hace que este deba fecharse época califal, lo que vendria confirmado por las abundantes estructuras de esta época que se han excavado con posterioridad en las proximidades.

En efecto, si relacionamos nuestra excavación con la efectuada en las proximidades del otro arroyo localizado en el área (SERRANO 1997), se nos confirma la existencia de una fase emiral-califal, que finalizaría con el abandono de toda el área periurbana y la concentración de la población en el interior del recinto urbano, al amparo de las murallas, ante el temor a los disturbios producidos por la fitna y la disgregación del califato en los reinos de Taifas y la consiguiente inestabilidad. Ese momento de abandono quedaría reflejado en la caida de las estructuras. Entonces, el terreno fue ocupado por el arroyo que selló en algunos puntos el habitat existente en época califal. No descartamos que la US 6 citada fuera el cauce del arroyo califal. 


\section{BIBLIOGRAFÍA}

AGUIRRE, J.; JIMÉNEZ, Ma C. (1979): Introducción al Jaén islámico. Jaén

AGUIRRE, J.; SALVATIERRA, V. (1989): "Cuando Jaén era Yayyan". Jaén VOL. II Historia. Granada.

LÁZARO, S. (1987): Las fuentes de Jaén. Jaén.
LÁZARO, S. (1988): Desarrollo histórico del casco urbano de Jaén hasta 1600. Jaén.

SALVATIERRA, V.; ALCÁZAR, E. (1996): "La distribución del agua en Jaén durante el período islámico" en Formas de Habitar e alimentaçao ena Idades Media. Mértola, 1993.

ULIERTE, Mª L. (1990): Jaén. La ciudad y su historia. Granada. 


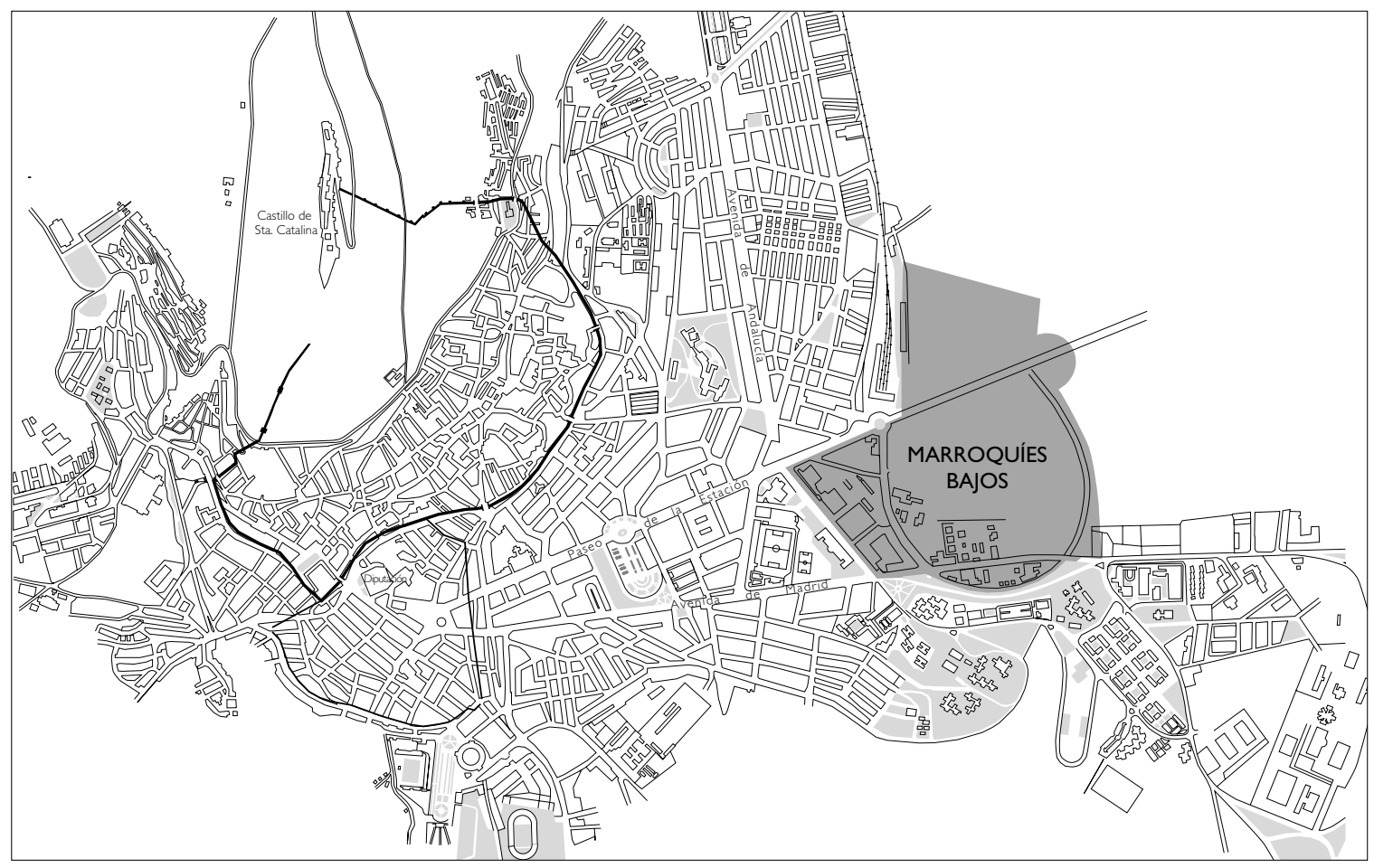

Fig. I. Plano de situación

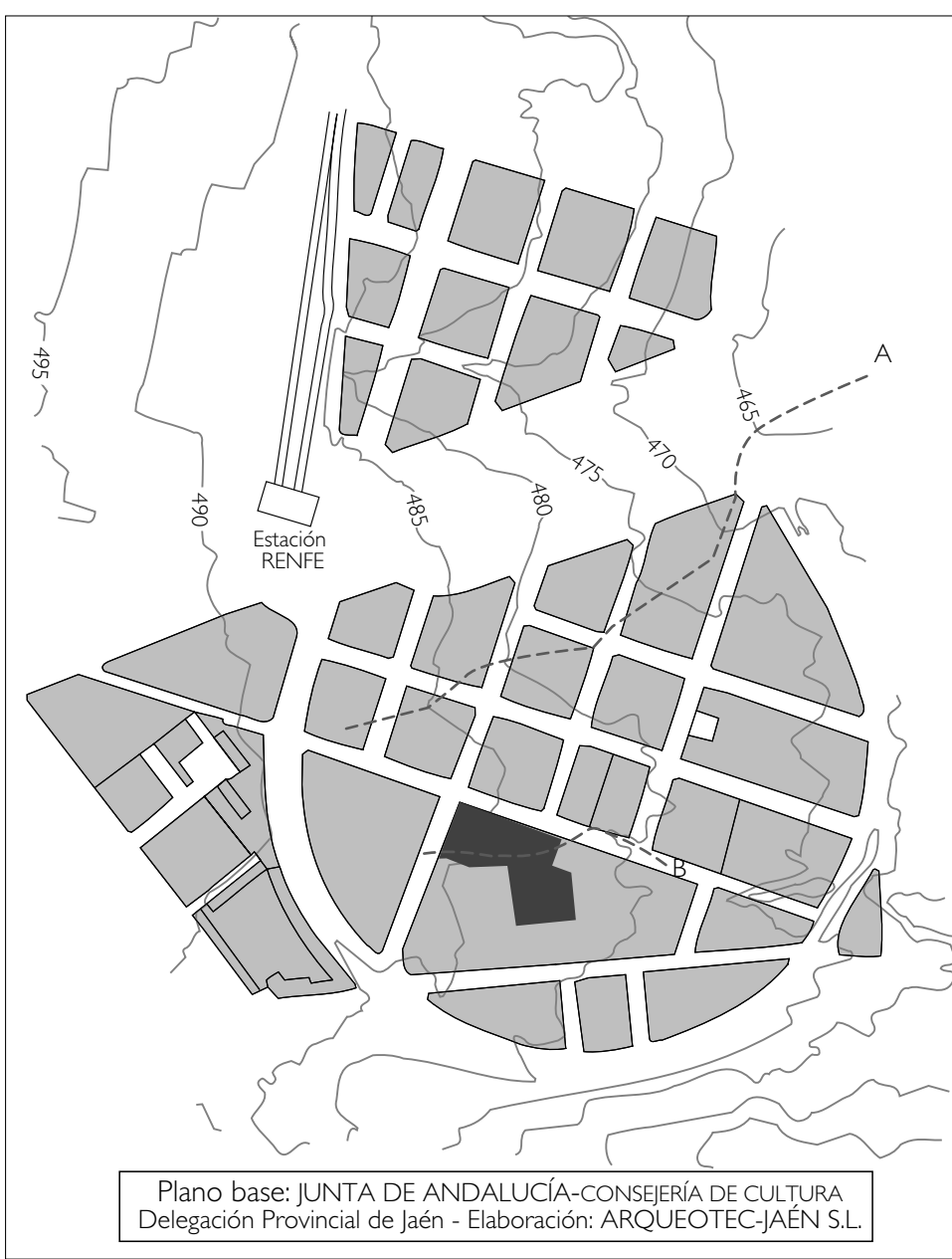

Fig. 2. Ubicación del solar 


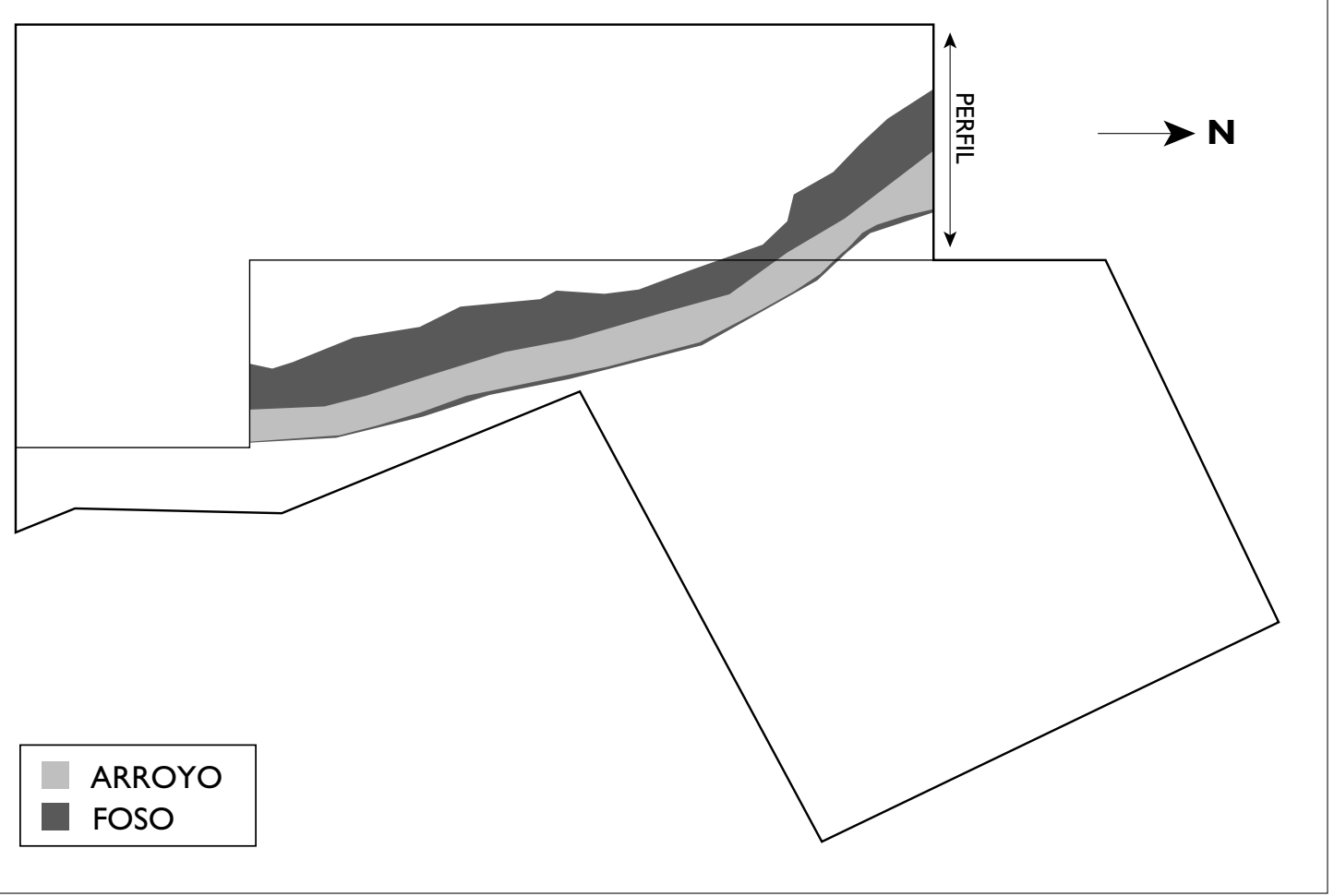

Fig. 3. Trazado del arroyo

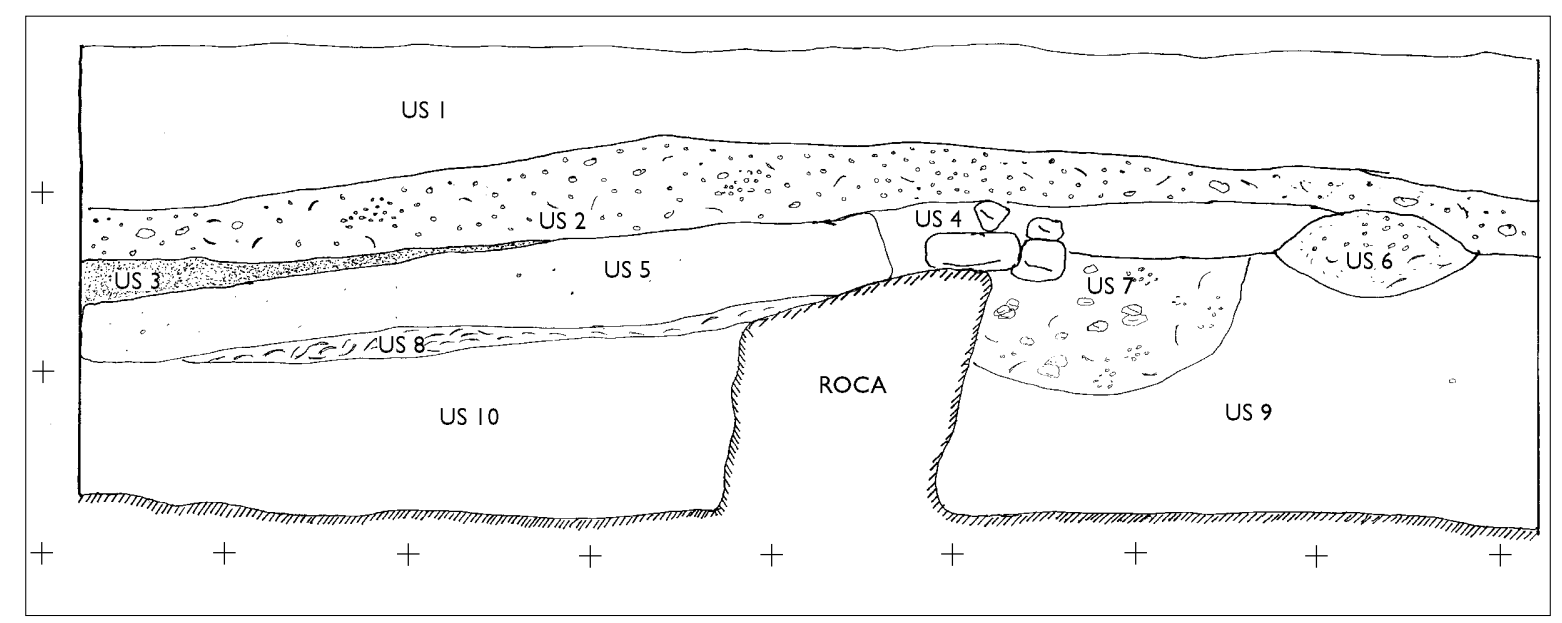

Fig. 4. Perfil Norte 